\title{
CHECKLIST E ABUNDÂNCIA DOS CNIDÁRIOS NOS AMBIENTES RECIFAIS DE MARAGOGI, ALAGOAS.
}

Recebido em: $17 / 09 / 2014$

Aceito em: 20/10/2014

\author{
Felipe Rafael Brasiliano CAVALCANTE ${ }^{1}$ \\ Sawana Caroline Aquino BORGES ${ }^{1}$ \\ Erika Flávia Crispim e SANTANA ${ }^{2}$ \\ Fernanda Duarte AMARAL ${ }^{3}$
}

\section{RESUMO}

O objetivo do trabalho foi 0 levantamento dos cnidários presentes nos recifes de Maragogi (Alagoas), além da análise do percentual de cobertura dos organismos bentônicos, para coleta de informações faunísticas básicas para o manejo e a conservação desses ecossistemas. O estudo foi realizado no período de dezembro de 2012 a julho de 2013, através de mergulhos livres, com a identificação e quantificação da porcentagem de cobertura dos organismos bentônicos, além do censo visual, utilizando o protocolo AGRRA Band e Line nos recifes das Taócas e
Galés. Os resultados evidenciaram através do percentual de cobertura, a predominância algal semelhante a outros locais do Nordeste do Brasil. Além disso, foram identificados dezessete táxons entre corais, octocorais. zoantídeos e hidróides calcários, sendo sete novos registros de ocorrência para os recifes de Maragogi. A predominância entre os cnidários foi de Millepora alcicornis, com cerca de $30 \%$ de cobertura em cada recife. Foram observados danos físicos e alterações nas colônias do referido organismo, causados por embarcações utilizadas no turismo e na pesca de subsistência.

Palavras-chave: Levantamento taxonômico, cnidários, conservação, ecossistema recifal, manejo.

\section{ABSTRACT}

The aim of this work was a checklist of cnidarian present on the reefs of Maragogi (Alagoas - Brazil), to collect basic faunal informations to management and conservation of these ecosystems. The study was conducted from December 2012 to July 2013, through free dives with the identification and percentage cover of benthic organisms quantification, beyond visual census using AGRRA Band and Line protocol on the reefs of Taócas and Galés. The results evidenced through the percentage of algal cover a similar prevalence to other places in the Northeast of Brazil. In addition, 17 taxa of corals, octocorals, zoanthids and calcified hydroids were identified, seven new occurrence records for Maragogi reefs. The most abundant cnidarian was Millepora alcicornis with about 30\% cover on each reef. Physical damage and changes in colonies caused by the anchors of local boats used for tourism and subsistence fishing were observed.

Key words: Checklist, cnidarians, conservation, reef ecosystem, management.

\section{INTRODUÇÃO}

Os ambientes recifais são ecossistemas costeiros e/ou oceânicos importantes, caracterizados pela grande abundância e riqueza de espécies, apresentando alta diversidade de organismos sésseis (REAKA-KUDLA, 1997; AMARAL et al. 2006). Além de fornecerem abrigo e suporte a uma variedade de espécies, esses ecossistemas também fornecem uma série de serviços e recursos para o homem, tendo, assim, importância não só ecológica, mas, também, econômica e social (MOBERG; FOLKE 1999; LEÃO; DOMINGUEZ 2000; AMARAL et al. 2007; KNOWLTON, 2008).

\footnotetext{
${ }^{1}$ Departamento de Oceanografia, Universidade Federal de Pernambuco.

E-mail: felipe_rbc@ibest.com.br; sawana_ufrpe@hotmail.com;

${ }^{2}$ Departamento de Zoologia, Universidade Federal da Paraíba. E-mail: erika.santana2@gmail.com;

${ }^{3}$ Departamento de Biologia, Universidade Federal Rural de Pernambuco. E-mail: fdamaral@db.ufrpe.br.
} 
A importância econômica dos recifes também é considerável, visto que estes são grandes atrativos turísticos, além de darem suporte e abrigo a uma variedade de comunidades marinhas de interesse econômico (MELO et al., 2005; COSTA et al., 2007). Entretanto, a expansão demográfica, as atividades pesqueiras excessivas, o aumento nas concentrações de nutrientes no oceano e o aquecimento global que se intensificaram ao longo das décadas, geraram um desequilíbrio na rede de interações específicas dentro e entre ecossistemas (HUGHES et al., 2003; GOLDBERG; WILKINSON, 2004; LESSER, 2007; MARKEY et al., 2007; MORA et al., 2007; MORA; GINSBURG, 2007).

Em relação aos problemas de desequilíbrios nos recifes, os organismos lá existentes respondem rapidamente às variações nos parâmetros físicos como a luminosidade e temperatura (GLYNN, 1993; CAVALCANTE; AMARAL, 2013), ou pela influência antrópica (SARMENTO; SANTOS, 2012). Sendo assim, a fauna recifal, especialmente, os corais e hidróides calcários, são caracterizados como ótimos bioindicadores dos impactos sobre os recifes (COOPER et al., 2009).

Áreas de conservação são de suma importância para manter o equilíbrio nos ecossistemas ameaçados. Em termos de litoral brasileiro, o número de áreas de conservação marinhas vem aumentando consideravelmente. Todavia, a distribuição das mesmas não é regular, deixando extensas áreas totalmente desprotegidas em muitos estados, como por exemplo, a área de aproximadamente $600 \mathrm{~km}$ entre o Arquipélago dos Abrolhos (BA) e a Reserva Extrativista Marinha de Arraial do Cabo (RJ) (MAIDA; FERREIRA, 2006).

A Área de Proteção Ambiental Costa dos Corais é considerada a maior unidade de conservação marinha brasileira e abrange $135 \mathrm{~km}$ da costa pernambucana e alagoana, sendo de grande importância ecológica, econômica e científica (MAIDA; FERREIRA, 2006; STEINER et al., 2006). Contudo, o conhecimento científico acerca desses ambientes no local ainda é muito escasso e a necessidade de preservação da biodiversidade recifal e gerenciamento dos recursos pesqueiros são urgentes.

Deste modo, o presente estudo realizou um levantamento dos antozoários e hidróides calcários presentes nos recifes de Maragogi (Alagoas). A avaliação da distribuição e composição faunística coralínea nos ambientes recifais fornece informações básicas de conhecimento para o manejo e a conservação desses ecossistemas.

\section{ÁREA ESTUDADA}

As coletas foram realizadas nos recifes das Taócas $\left(9^{\circ} 1^{\prime} 21.3^{\prime \prime} \mathrm{S}\right.$ e $\left.35^{\circ} 11^{\prime} 36.6^{\prime \prime} \mathrm{W}\right)$ e das Galés (902'37"S e $25^{\circ} 11^{\prime} 31,6^{\prime \prime} \mathrm{W}$ ) da cidade de Maragogi (Fig.1), pertencentes à Área de Proteção Ambiental Costa dos Corais que se estende desde o Estado de Pernambuco, a partir dos recifes do município de Tamandaré, até o Estado de Alagoas (MAIDA; FERREIRA, 2006). Tal localidade possui um clima megatérmico subúmido, temperatura média de $26^{\circ} \mathrm{C}$ e pluviosidade anual em torno de $1300 \mathrm{~mm}$ (CPRM, 2005).

Os ambientes recifais das Taócas e Galés possuem áreas abertas para a visitação e áreas fechadas. O turismo local transporta cerca de 60 mil visitantes por ano para esses recifes, com picos de cerca de 800 visitantes por dia (FERREIRA; MAIDA, 2006), através de catamarãs e/ou embarcações adaptadas/improvisadas, que eram anteriormente utilizadas para pesca (Observação pessoal).

\section{MATERIAL E MÉTODOS}

O estudo foi realizado durante os meses de dezembro de 2012 a julho de 2013, utilizando - Protocolo AGRRA (Atlantic and Gulf Rapid Reef Assessment Project) Band e Line (AGRRA, 2010). Para cada recife (Taócas e Galés), foram realizados seis transectos de $10 \mathrm{~m}$ para a análise Line, com a identificação e porcentagem de cobertura dos organismos bentônicos e seis transectos de $10 \times 1 \mathrm{~m}$ (comprimento x largura) para a análise Band, quantificando o número de colônias quebradas e inteiras de Millepora alcicornis, além do método de censo visual para uma busca intensiva de organismos que não foram visualizados nos transectos. 
A maioria dos dados amostrados foi obtida na região do infralitoral e apenas no mediolitoral quando a plataforma recifal estava completamente exposta na baixa-mar. A profundidade dos recifes no período de coleta variou de 0 a 3 metros.

Para a análise da contribuição de cada categoria de organismos no recobrimento do local estudado, analisaram-se os índices de frequência relativa, percentagem de cobertura (taxonomia dominante) e importância, através das fórmulas: Índice de frequência relativa (Fr): $\mathrm{Fr}=\mathrm{Fi} / \mathrm{Ft} \times 100\left(\mathrm{Fi}=\mathrm{N}^{\circ}\right.$ de transectos em que a espécie ocorre e $\mathrm{Ft}=\mathrm{N}^{\circ}$ total de transectos $)$ e Índice de importância $(\mathrm{I}): \mathrm{I}=\mathrm{Fr} \times \mathrm{Td} / 100(\mathrm{Fr}=$ frequência relativa $\mathrm{e} \mathrm{Td}=$ percentagem de cobertura).

A partir dos índices descritores citados, as amostras de cada transecto foram agrupadas e a comunidade foi analisada como um todo. Esses agrupamentos seguem o trabalho realizado por Villaça; Pitombo (1997) com adaptação para o Protocolo AGRRA.

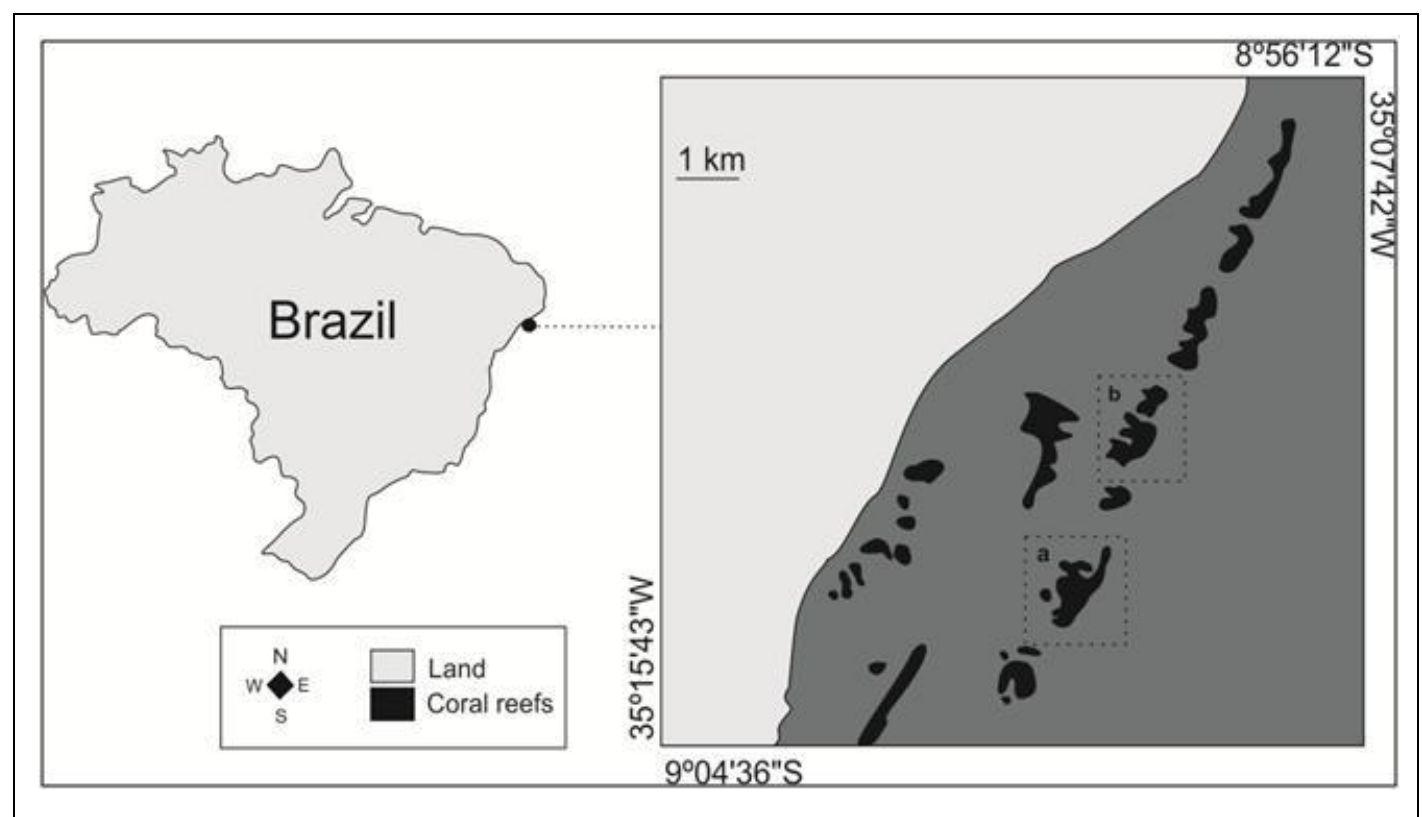

Figura 1 - Mapa dos recifes das Galés (a) e Taócas (b) de Maragogi, adaptado de Feitosa et al. (2012).

\section{RESULTADOS E DISCUSSÃO}

Foi encontrado o total de dezessete táxons dentre corais, octocorais, zoantídeos e hidróides calcários. As espécies encontradas de corais escleractínios foram: Siderastrea stellata Verrill, 1868, Favia gravida Verrill, 1868, Mussismilia hispida (Verrill, 1868), Porites astreoides Lamarck, 1816, Agaricia agaricites (Linnaeus, 1758), Monstastraea cavernosa (Linnaeus, 1767) (Fig. 2a), Mussismilia harttii (Verrill, 1867) (Fig. 2b), Madracis decactis (Lyman, 1859); os octocorais Muriceopsis sp. (Fig. 2e), Plexaurella grandiflora Verrill, 1912 (Fig. 2f); os zoantídeos Palythoa caribaeorum (Duchassaing \& Michelotti, 1860), Zoanthus sociatus (Ellis, 1768), Zoanthus sp. e Protopalythoa variabilis (Duerden, 1898). Além disso, foram observados os hidróides calcários: Millepora alcicornis Linnaeus, 1758 (Fig. 2c e d) e Millepora braziliensis (Verrill, 1868).

Os resultados do AGRRA Line mostraram que a maior porcentagem de cobertura é de algas epilíticas (ALG - 49,2\%) seguida do hidróide calcário Millepora alcicornis (29,6\%) nos recifes das Taócas. Nos recifes das Galés os resultados foram semelhantes, sendo os organismos de maior percentual de cobertura as algas epilíticas $(39,7 \%)$, seguido do hidróide calcário M. alcicornis (29,8\%) (Fig. 3). 


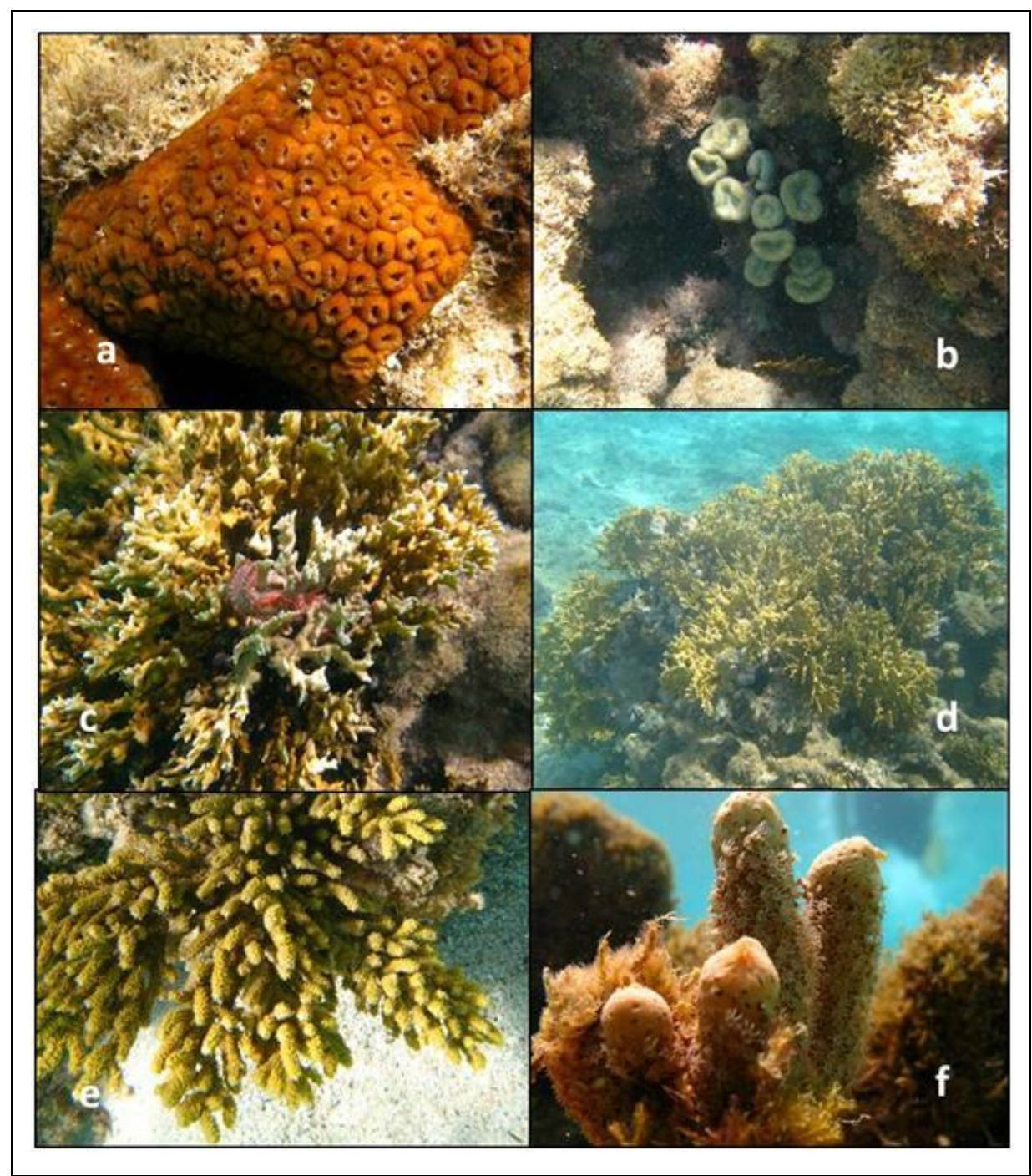

Figura 2 - Fotos da fauna coralínea dos recifes de Maragogi (Alagoas); a- Montastraea cavernosa, b- Mussismilia harttii, c- Millepora alcicornis e o anelídeo Hermodice carunculata, d-M. alcicornis, e- Muriceopsis sp., f- Plexaurella grandiflora. Fotos: Renato Correia.

Pitombo et al. (1988) encontraram $30 \%$ de cobertura de coral nos recifes de franja de Siriba, em Abrolhos (Bahia), além da presença de algas Corallinacea. Francini-Filho et al. (2013) também observaram alta cobertura de corais escleractínios nas paredes de um dos recifes estudados em Abrolhos, porém também observaram uma alta cobertura de macroalgas e algas epilíticas nesses recifes. Já nos recifes de Porto de Galinhas (Pernambuco), Barradas et al. (2010) verificaram o percentual do total de cobertura de cerca de $54 \%$ de macroalgas e de apenas $7,2 \%$ de corais e hidróides calcários, utilizando outro método de análise de cobertura com transectos. Esses resultados corroboraram com os resultados do presente estudo, no qual encontrou grande dominância de algas nos recifes. Diversos estudos apontam que a dominância de espaço por macroalgas e algas epilíticas impedem a cobertura de corais, prejudicando-os através da competição por espaço e por não permitir o recrutamento de larvas de corais (MCCOOK et al., 2001; BIRRELL et al., 2005; ARNOLD et al., 2010). Por isso, a relação entre corais e algas do local de estudo deve ser verificada de forma cuidadosa para avaliar suas interações e implicações no crescimento e degradação dos cnidários bentônicos.

A alta cobertura do hidróide calcário M. alcicornis evidencia um ambiente que possui características dos recifes brasileiros mostradas nos trabalhos de Laborel $(1969,1970)$ que se caracteriza por uma forte presença dessa espécie, criando as chamadas "zonas de Milleporas". Como os recifes de Maragogi exibem uma fragmentação, ou seja, não são contínuos, esse pode 
ser o principal fator para essa espécie apresentar uma grande cobertura, pois esses indivíduos geralmente tem uma afinidade pelo crescimento nas bordas dos recifes, de acordo com Lewis (1989).

Além disso, de acordo com Coni et al. (2012), M. alcicornis é refúgio para diversas espécies de peixes recifais. Nos recifes de Maragogi há 31 espécies de peixes recifais apresentando maior abundância para a espécie de peixe donzela Stegastes fuscus (FEITOSA et al., 2012). Coni et al. (2012), Pereira et al. (2012) e Leal et al. (2013) mostraram uma forte associação dessa espécie de peixe com esse hidróide calcário no Nordeste do Brasil, bem como associação com outras espécies. Tal fato implica na importância deste hidrocoral nos ambientes recifais de Maragogi.

A análise da frequência relativa (Tab. 1) nos recifes das Taócas mostra que os organismos mais observados nos transectos foram as algas epilíticas e o hidróide M. alcicornis com $100 \%$. Esses organismos apresentaram o maior índice de importância nesse recife. Em contrapartida, os organismos com frequências mais baixas foram as macroalgas e os zoantídeos, apresentando 16,66 \% ambos.

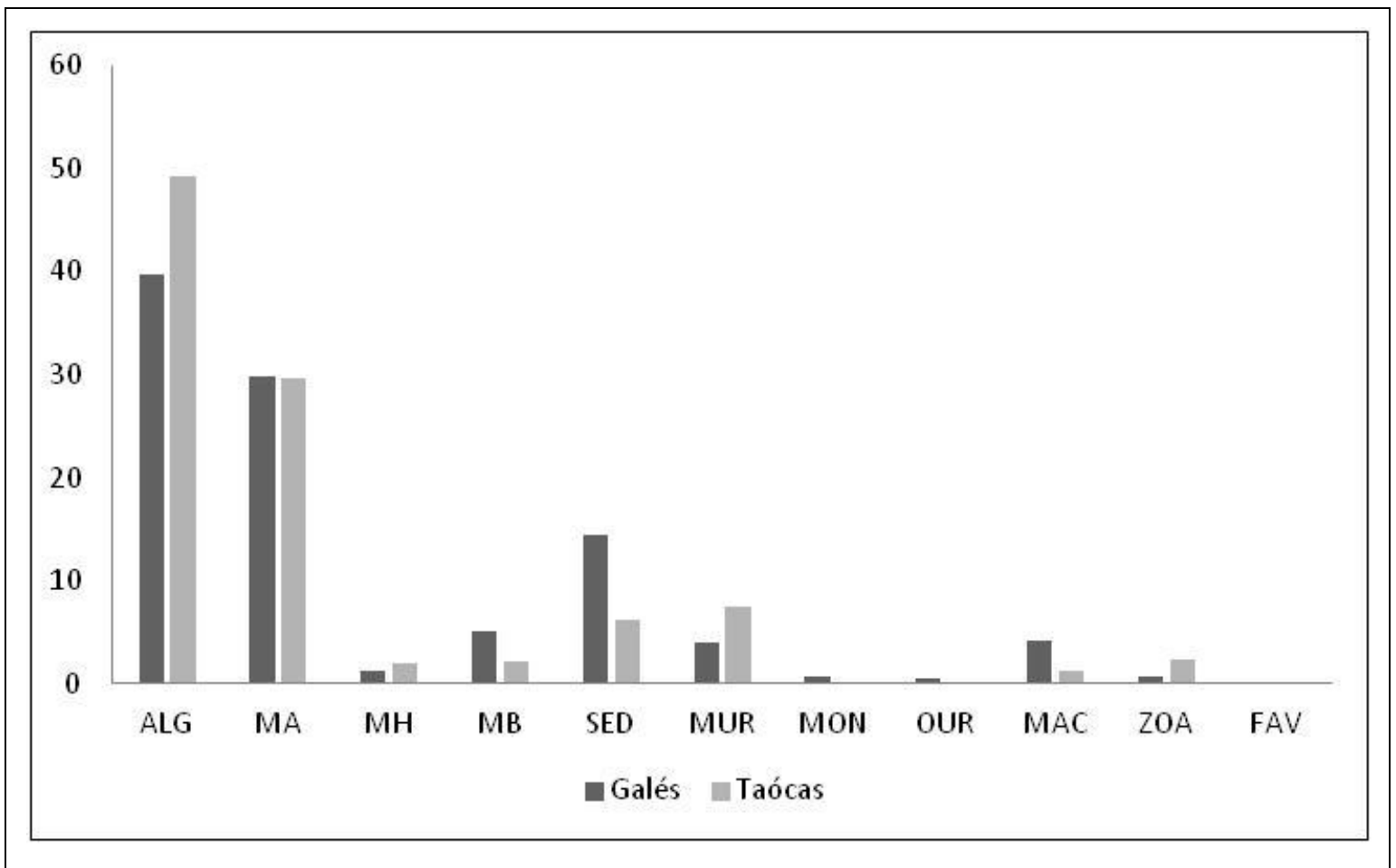

Figura 3 - Porcentagem de cobertura dos organismos nos recifes das Galés e Taócas obtida através do Protocolo AGRRA Line. ALG= algas epilíticas; MA= Millepora alcicornis; $\mathrm{MH}=$ Mussismilia harttii; $\mathrm{MB}=$ Millepora braziliensis; $\mathrm{SED}=$ sedimento; $\mathrm{MUR}=$ Muriceopsis sp.; MON= Montastraea cavernosa; OUR= ouriço; MAC= macroalga sp.; ZOA= zoantídeo; FAV= Favia gravida.

Nos recifes das Galés observou-se um padrão de frequência semelhante aos recifes das Taócas, onde os organismos mais visualizados foram algas epilíticas e o hidróide M. alcicornis com $100 \%$. Comunidades recifais brasileiras importantes como os recifes de Abrolhos são dominados por algas, geralmente algas filamentosas, além de ser encontrado o mesmo padrão para outros recifes no mundo (HATCHER; LARKUM, 1983; KLUMPP; MCKINNON, 1992; PITOMBO; VILLAÇA, 1996).

A cobertura de algas epilíticas sugere uma grande capacidade de fixação de nitrogênio de acordo com Wiebe et al. (1975). Hatcher (1990) cita que a herbivoria e a quantidade de nutrientes influenciam diretamente na biomassa vegetal nos recifes de corais. Além disso, segundo Littler et al. (1983), esses fatores são os maiores controladores dos produtores 
primários bentônicos, como, por exemplo, corais e algas (filamentosas, frondosas e macroalgas).

Tabela 1 - Análise da frequência relativa e índice de importância das diferentes categorias encontradas no levantamento dos transectos. $A L G=$ algas epilíticas; $M A=$ Millepora alcicornis; $\mathrm{MH}=$ Mussismilia harttii; $\mathrm{MB}=$ Millepora braziliensis; $\mathrm{SED}=$ sedimento; MUR= Muriceopsis sp.; MON= Montastraea cavernosa; OUR= ouriço; $\mathrm{MAC}=$ macroalgas; $\mathrm{ZOA}=$ zoantídeo; FAV= Favia gravida .

\begin{tabular}{lllll}
\hline \multirow{2}{*}{ Categorias } & \multicolumn{2}{l}{ Taócas } & \multicolumn{3}{c}{ Galés } \\
\cline { 2 - 5 } & Fr & I & Fr & I \\
ALG & 100 & 49,23 & 100 & 39,66 \\
MA & 100 & 29,56 & 100 & 29,76 \\
MUR & 83,33 & 6,20 & 50 & 1,95 \\
MH & 50 & 0,98 & 33,33 & 0,38 \\
ZOA & 16,66 & 0,40 & 33,33 & 0,24 \\
MB & 50 & 1,03 & 83,33 & 4,16 \\
MAC & 16,66 & 0,19 & 33,33 & 1,35 \\
MON & - & - & 33,33 & 0,25 \\
OUR & - & - & 16,66 & 0,06 \\
FAV & - & - & 16,66 & 0,02 \\
\hline
\end{tabular}

Os organismos Agaricia agaricites, Siderastrea stellata, Favia gravida, Palythoa caribaeorum, Zoanthus sociatus, Plexaurella grandiflora e Muriceopsis sp. foram novas ocorrências nos recifes de Maragogi. As espécies citadas anteriormente não foram observadas nos estudos com "Reef Check" de Ferreira; Maida (2006), quando houve registros de apenas seis espécies entre corais e hidróides calcários observados.

Apesar de M. alcicornis ser a espécie de hidróide calcário mais encontrada dentre outros organismos construtores dos recifes, a análise a partir dos resultados obtidos pelo AGRRA Band, verificou que cerca de $50 \%$ das colônias estavam quebradas (Figs. 4 e 5). Isso é resultante em grande parte das âncoras dos barcos que se fixam nas bordas dos recifes e da falta do controle do turismo nos recifes de Maragogi (observação pessoal), demonstrando, em alguns transectos, maior quantidade de colônias quebradas do que inteiras.

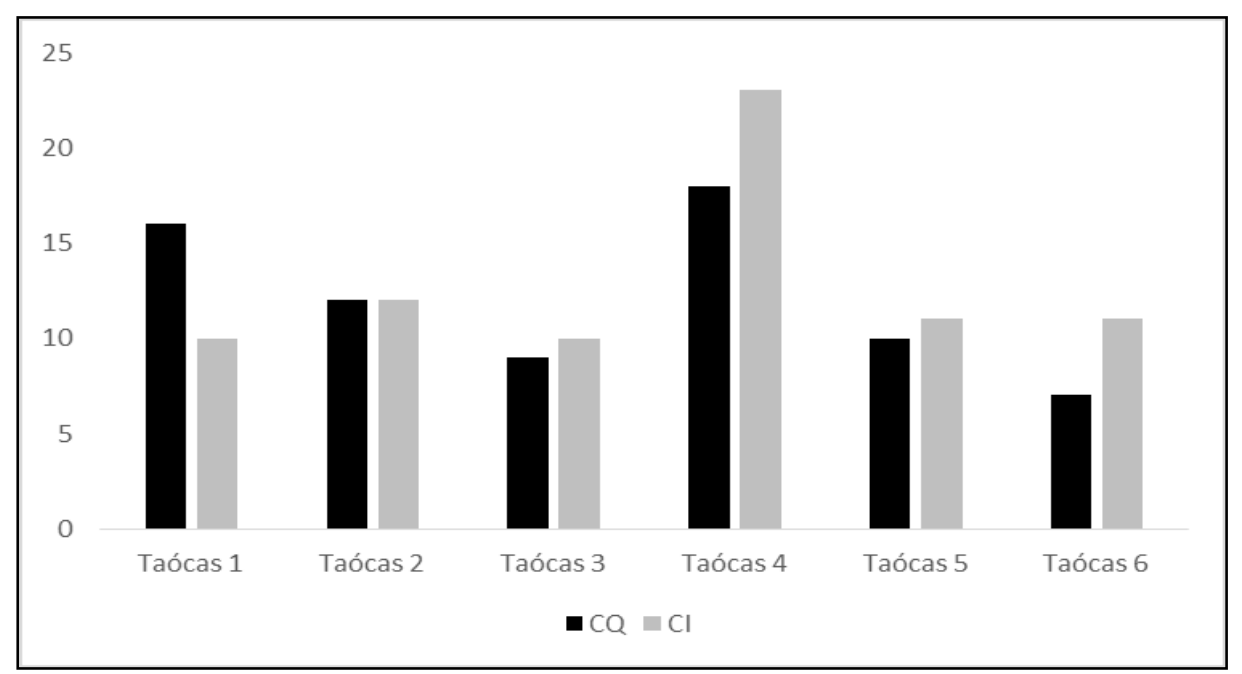

Figura 4 - Número de colônias quebradas e inteiras de Millepora alcicornis, através do AGRRA Band nos recifes das Taócas (Maragogi - Alagoas). $\mathrm{CQ}=$ colônia quebrada; $\mathrm{CI}=$ colônia inteira. 


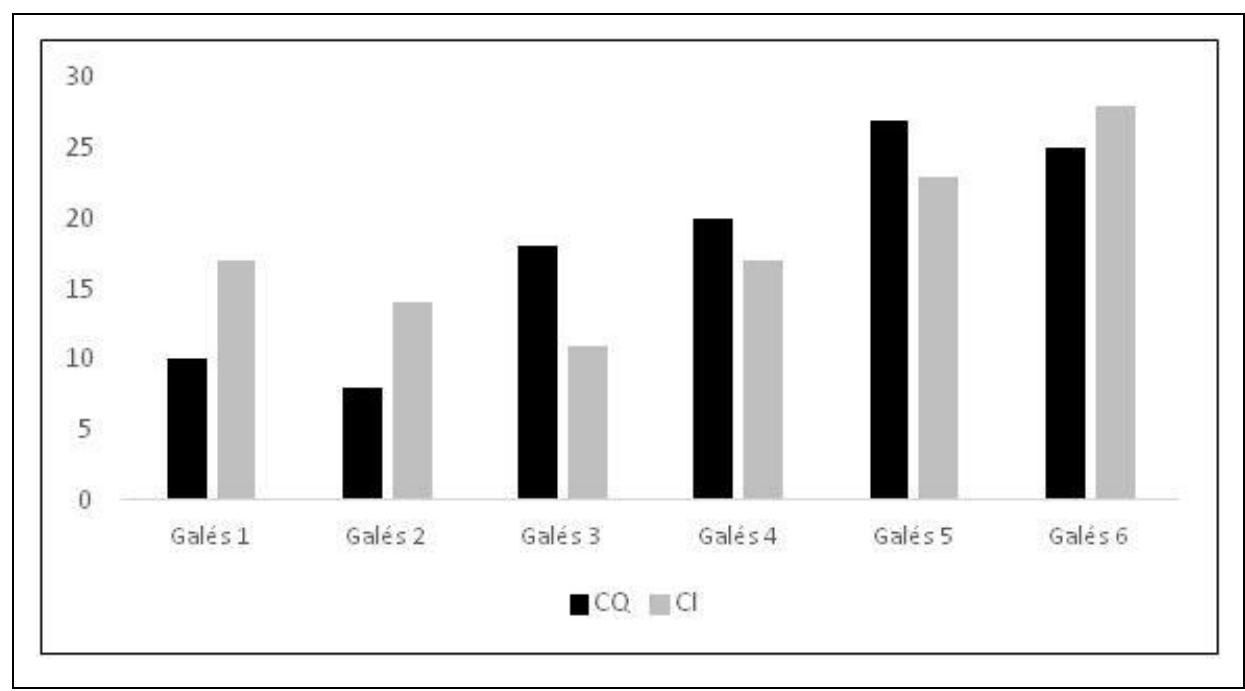

Figura 5 - Número de colônias quebradas e inteiras de Millepora alcicornis analisada através do AGRRA Band nos recifes das Galés (Maragogi - Alagoas). CQ= colônia quebrada; $\mathrm{CI}=$ colônia inteira.

Alguns dos maiores impactos descritos aos ambientes recifais relacionados com o turismo são a prática do mergulho, de acordo com Medio et al. (1997), Hawkins et al. (1999) e Tratalos; Austin (2001); a ancoragem (DISNDALE; HARRIOT, 2004) e o pisoteio (RODGERS; COX, 2003). Além da contribuição social e econômica gerada pela interação entre o turista e a comunidade que sobrevive desse fator, a má administração de órgãos fiscalizadores ou a ausência de projetos concretos de monitoramento desses recifes dificultam a observação dos danos gerados por tais atividades.

Uma das praias do Nordeste que também sofre com o turismo é a Praia de Porto de Galinhas (PE), pois de acordo com Barradas et al. (2012), essa apresenta áreas que sofreram impactos com o turismo descontrolado nos ambientes recifais. A associação de impactos negativos do turismo foi visualizada a partir da análise de nutrientes que mostraram altas concentrações de amônia no verão (alta estação do turismo) de acordo com os referidos. Tais fatos induzem a conclusão de que o turismo em recifes do Nordeste do Brasil é um importante fator que pode contribuir com o desequilíbrio nos recifes.

\section{CONCLUSÕES}

O estudo foi capaz de registrar, através do percentual de cobertura, uma predominância algal semelhante a outros locais do Nordeste do Brasil. Além disso, foram identificados dezessete táxons de corais, octocorais e hidróides calcários, sendo sete novos registros de ocorrência para os recifes de Maragogi.

A maior predominância dentre os cnidários foi a de $M$. alcicornis, com cerca de $30 \%$ de cobertura em cada recife. Contudo, em alguns pontos a quantidade de colônias inteiras foi a mesma de colônias quebradas. Tal fato ajudou a identificar danos físicos e alterações nas colônias causadas por embarcações locais que se utilizam do turismo e pesca para sobrevivência. Portanto, faz-se necessário um controle e monitoramento maior das embarcações acerca do manuseio dos recifes para o turismo, pois tais impactos podem influenciar negativamente na saúde dos organismos recifais.

\section{AGRADECIMENTOS}

Ao DOCEAN/UFPE (Departamento de Oceanografia da Universidade Federal de Pernambuco), a CAPES e CNPq pelo fornecimento das bolsas para os autores e à oceanógrafa Camila Brasil pela ajuda na metodologia realizada. 


\section{REFERÊNCIAS BIBLIOGRÁFICAS}

AGRRA. Atlantic and Gulf Rapid Reef Assessment. AGRRA PROTOCOLS VerSION 5.4. Revision by Judith C. Lang, Kenneth W. Marks, Philip A. Kramer, Patricia Richards Kramer, and Robert N. Ginsburg. Link: http://www.agrra.org/. Acessado em: 03 de fevereiro de 2014 às 03:38. 2010.

AMARAL, F. D.; HUDSON, M. M.; STEINER, A. Note on the widespread bleaching observed at the Manuel Luiz Marine State Park, Maranhão, Brazil. Arquivos de Ciências do Mar, v. 39, p. 138- 141. 2006.

AMARAL, F. D.; HUDSON, M. M.; STEINER, A. Q.; RAMOS, C.A.C. Corals and calcified hydroids of the Manuel Luiz Marine State Park (State of Maranhão, Northeast Brazil). Biota Neotropica, v.7, n.3. 2007.

ARNOLD, S. N.; STENECK, R. S.; MUMBY, P. J. Running the gauntlet: inhibitory effects of algal turfs on the processes of coral recruitment. Marine Ecology Progress Series, v. 414, p. 91105. 2010.

BARRADAS, J. I.; AMARAL, F. M. D. ; HERNANDEZ, M. I. M. ; FlORES, M. ; STEINER, A. Q. Spatial distribution of benthic macroorganisms on reef flats at Porto de Galinhas Beach (northeastern Brazil), with special focus on corals and calcified hydroids. Biotemas (UFSC), $v$. 23, p. 1-11. 2010.

BARRADAS, J. I.; AMARAL, F. D.; HERNÁNDEZ, M. I.; FLORES-MONTES, M. J.; STEINER, A. Q. Tourism impact on reef flats in Porto de Galinhas beach, Pernambuco, Brazil. Arquivo de Ciências do Mar, v. 45, n. 2, p 81-88. 2012.

BIRRELL, C.L.; MCCOOK, L.J. \& WILLIS, B.L. Effects of algal turfs and sediment on coral settlement. MarinePollution Bulletin, v. 51, p. 408-414. 2005.

CAVALCANTE, F.R.B.; AMARAL, F.D. Variação das zooxantelas e branqueamento no hidróide calcário Millepora alcicornis Linnaeus, 1758 nos recifes de Porto de Galinhas - Nordeste brasileiro. Tropical Oceanography, v. 42, p.1-11. 2013.

CONI, E. ; FERREIRA, C. M.; MOURA, R.L.; MEIRELES, P.M.; KAUFMAN, L.; FRANCINI-FILHO, R.B. An evaluation of the use of branching fire-corals (Millepora spp.) as refuge by reef fish in the Abrolhos Bank, eastern Brazil. Environmental Biology of Fishes, v. 94, p.1-11. 2012.

COOPER, T. F.; GILMOUR, J. P.; FABRICIUS, K. E. Bioindicators of changes in water quality on coral reefs: review and recommendations for monitoring programmes. Coral reefs, v. 28, p.589-606. 2009.

COSTA, C. F.; SASSI, R.; COSTA M. A. J. ; BRITO A. C. L. de. Recifes costeiros da Paraíba, Brasil: usos, impactos e necessidades de manejo no contexto da sustentabilidade. Gaia Scientia, v.1, n.1, p. 37-45. 2007.

CPRM. Serviço Geológico do Brasil. Projeto cadastro de fontes de abastecimento por água subterrânea. Diagnóstico do município de Maragogi estado de Alagoas. Org. MASCARENHAS J. C.; BELTRÃO, B. A.; JUNIOR , L. C. de S.. Recife: CPRM/PRODEEM, 12p. 2005.

DINSDALE, E. A.; HARRIOTT, V.J. Assessing An- chor Damage on Coral Reefs: A Case Study in Selection of Environmental Indicators. Environmental Management, v. 33, n. 1, p. 126-139. 2004.

FEITOSA, C. V.; CHAVES, L. de C. T.; FERREIRA, B. P.; de ARAÚJO, M. E. Recreational fish feeding inside Brazilian MPAs: impacts on reef fish community structure. Journal of the Marine Biological Association of the United Kingdom, v. 1, p. 1-9. 2012.

FERREIRA, B. P; MAIDA, M. Monitoramento dos Recifes de Coral do Brasil: Situação Atual e Perspectivas. Ministério do Meio Ambiente - MMA. 250 p. 2006.

FRANCINI-FILHO, R.B.; CONI, E.C.O.; MEIRELLES, P.M.; AMADO-FILHO, G.M.; THOMPSON, F.L.; PEREIRA-FILHO, G.H.; BASTOS, A.C.; ABRANTES, D.P.; FERREIRA, C.M.; GIBRAN, F.Z.; 
GUTH, A.Z.; SUMIDA, P.YG.; OLIVEIRA, N.L.; KAUFMAN, L.; MINTE-VERA, C.M.; MOURA, R.L. Dynamics of coral reef benthic assemblages of the Abrolhos Bank, Eastern Brazil: Inferences on natural and anthropogenic drivers. PLoS One, v. 8, n. 1. 2013.

GLYNN, P. W. Coral reef bleaching: Ecological perspectives. Coral reefs, v. 12, p. 1-17. 1993.

GOLDBERG, J.; WILKINSON, C. Global threats to coral reefs: coral bleaching, global climate change, disease, predator plagues, and invasive species. In: WILKINSON, C. (Ed.) Status of Coral Reefs of the World. Australian Institute of Marine Science, Townsville, Australia. p. 6792. 2004.

HATCHER, B.G. Coral reef productivity: A hierarchy of pattern and process trends. Ecology and Evolution, v.5, p.149-155. 1990.

HATCHER, B. G.; LARKUM, A. W. D. An experimental analysis of factors controlling the standing crop of the epilithic algal community on a coral reef. Journal of Experimental Marine Biology and Ecology, v. 69, p. 61- 84. 1983.

HAWKINS, J. P., C. M. ROBERTS, T. VAN'T HOF, K. DE MEYER, J. TRATALOS, AND C. ALDAM. . Effects of recreational scuba diving on Caribbean coral and fish communities. Conservation Biology, v.13, p. 888-897. 1999.

HUGHES, T. P.; BAIRD, A. H.; BELLWOOD, D. R.; CARD, M.; CONNOLLY, S. R.;FOLKE, C.; GROSBERG, R.; HOEGH-GULDBERG, O.; JACKSON, J. B. C.; KLEYPAS, J.; LOUGH, J. M.; MARSHALL, P.; NYSTRÖM, M.; PALUMBI, S. R.; PANDOLFI, J. M.; ROSEN, B.; ROUGHGARDEN, J. Climate Change, Human Impacts, and the Resilience of Coral Reefs. Science, v. 301, p. 929933. 2003.

KLUMPP, D. W.; MCKINNON, A. D. Community structure, biomass and productivity of epilithic algal communities on the Great Barrier Reef: dynamics at different spatial scales. Marine Ecology Progress Series, v.86, p.77-89. 1992.

KNOWLTON, N. Coral reefs. Current Biology, v.18, n. 1, p. 18-21, 2008.

LABOREL, J. Les peuplements de madreporaires dês cotes tropicales du Brésil. Annales Universitê d'Abidjan, Serie E, v. 2, n. 3, p.1-261. 1969.

LABOREL, J. Madréporaries et hydrocoralliaires récifaux des côtes brésiliennes. systématique, écologie, répartition verticale et géographique. Résultats Scientifiques des campagnes, Calypso, v. 9, n. 25, p.171-229. 1970.

LEAL, I. C. S; Pereira, P. H. C.; Araújo, M. E. de. Coral reef fish association and behaviour on the fire coral Millepora spp. in north-east Brazil. Journal of the Marine Biological Association of the United Kingdom, v. 93, n. 6, 1703-1711. 2013.

LEÃO, Z. M. A. N.; DOMINGUEZ, J. M. L. Tropical coast Brazil. Marine Pollution Bulletin, v. 41, p. $112-122,2000$.

LESSER, M. P. Coral reef bleaching and global climate change: Can corals survive the next century? Proceedings of the National Academy of Sciences, v. 104, n. 13, p. 5259-5260, 2007.

LEWIS, J. B. The ecology of Millepora: A review. Coral reefs, v.8, n.3, p.99- 107, 1989.

LITTLER, M. M.; LITTLER, D. S.; TAYLOR, P. R. Evolutionary strategies in a tropical barrier reef system: functional-form groups of marine macroalgae. Journal of Phycology, v.19, p. 229237. 1983.

MCCOOK, L.J.; JOMPA, J. \& DIAZ-PULIDO, G. Competition between corals and algae on coral reefs: a review of evidence and mechanisms. Coral Reefs, v. 19, p.400-417. 2001.

MAIDA, M.; FERREIRA, B. P. Área de Proteção Ambiental Costa dos Corais. In: PRATES, A. P. L. (Ed.) Atlas dos recifes de corais nas unidades de conservação brasileiras. Brasília: Instituto Brasileiro do Meio Ambiente e dos Recursos Naturais Renováveis- IBAMA/MMA, p. 87106. 2006. 
MARKEY, K. L.; BAIRD, A. H.; HUMPHREY, C.; NEGRI, A. P. Insecticides and a fungicide affect multiple coral life stages. Marine Ecology Progress Series, v. 330, p.127-137. 2007.

MEDIO, D.; ORMOND, R. F. G.; PEARSON, M. Effect of briefings on rates of damage to corals by scuba divers. Biological Conservation, v.79, p. 91-95. 1997.

MELO, R. de S.; CRISPIM, M. C.; LIMA, E. R. V. de. Turismo em ambientes recifais: em busca da transição para sustentabilidade. Caderno Virtual de Turismo, v.5, n.4, p.34-42. 2005.

MOBERG, F.; FOLKE, C. Ecological goods and services of coral reef ecosystems. Ecological Economics, Amsterdam, v. 29, p. 215-233. 1999.

MORA, C.; GINSBURG, R. A clear human footprint in the coral reefs of the Caribbean. Proceedings of the Royal Society. p. 1-8. 2007.

MORA, C.; METZKER, R.; ROLLO, A.; MYERS, R. A. Experimental simulations about the effects of habitat fragmentation and overexploitation on populations facing environmental warming. Proceedings of the Royal Society, v.274, p. 1023-1028. 2007.

PEREIRA, P. H. C; LEAL, I. C. S.; ARAÚJO, M. E. de; SOUZA, A. T. Feeding association between reef fishes and the fire coral Millepora spp. (Cnidaria: Hydrozoa). Marine Biodiversity Records, v. 5, n. 42, p. 1-4. 2012.

PITOMBO, F. B.; RATTO, C. C.; BELÉM, M. J. C. Species diversity and zonation pattern of hermatypic coraIs at two fringing reefs of Abrolhos Archipelago, Brazil. In: Proceedings of the 6th International Coral Reef Symposium, v. 2, p. 817-820. 1988.

REAKA-KUDLA, M. L. The global biodiversity of coral reefs: A comparison with rain forests. In: REAKA-KUDLA, M. L.; WILSON, D. O.; WILSON, E. O. (Eds.) Biodiversity II. Understanding and protecting our biological resources. Washington: Joseph Henry Press, p. 83-108. 1997.

RODGERS, K.S; COX, E.F. The effects of trampling on Hawaiian corals along a gradient of human use. Biological Conservation, v.112, p. 383-389. 2003.

SARMENTO, V. C.; SANTOS, P. J. P. Trampling on coral reefs: tourism effects on harpacticoid copepods. Coral Reefs, v. 31, p. 135-146. 2012.

STEINER, A. Q.; ELOY, C. C.; AMARAL, J. R.; AMARAL, F. M. D.; SASSI, R. O turismo em áreas de recifes de coral: considerações acerca da Área de Proteção Ambiental Costa dos Corais (Estados de Pernambuco e Alagoas). OLAM (Rio Claro), v. 6, n. 2, p. 281-296. 2006.

TRATALOS, J.; AUSTIN, T. 2001. Impacts of recreational SCUBA diving on coral com- munities of the Caribbean island of Grand Cayman. Biological Conservation, v. 102, p. 67-75. 2001

VILLAÇA, R.; PITOMBO, F.B. Benthic communities of shallow-water reefs of Abrolhos, Brazil. Revista Brasileira de Oceanografia, v. 45, p. 35-43. 1997.

WIEBE, W. J.; JOHANES, R. E.; WEBB, K. L. Nitrogen fixation in a coral reef community. Science, v. 188, p. 257-259. 1975. 